\title{
THERMAL MODEL CALIBRATION FOR MINOR PLANETS OBSERVED WITH WISE/NEOWISE: COMPARISON WITH INFRARED ASTRONOMICAL SATELLITE
}

\author{
A. Mainzer ${ }^{1}$, T. Grav ${ }^{2}$, J. Masiero $^{1}$, J. Bauer $^{1,3}$, E. Wright $^{4}$, R. M. Cutri ${ }^{3}$, R. WAlker $^{5}$, And R. S. McMillan $^{6}$ \\ ${ }^{1}$ Jet Propulsion Laboratory, California Institute of Technology, Pasadena, CA 91109, USA; amainzer@jpl.nasa.gov \\ ${ }^{2}$ Department of Physics and Astronomy, Johns Hopkins University, Baltimore, MD 21218, USA \\ ${ }^{3}$ Infrared Processing and Analysis Center, California Institute of Technology, Pasadena, CA 91125, USA \\ ${ }^{4}$ UCLA Astronomy, P.O. Box 91547, Los Angeles, CA 90095-1547, USA \\ ${ }^{5}$ Monterey Institute for Research in Astronomy, Monterey, CA, USA \\ ${ }^{6}$ Lunar and Planetary Laboratory, University of Arizona, 1629 East University Blvd., Kuiper Space Science Bldg. \#92, Tucson, AZ 85721-0092, USA \\ Received 2011 April 29; accepted 2011 June 23; published 2011 July 19
}

\begin{abstract}
With thermal infrared observations detected by the NEOWISE project, we have measured diameters for 1742 minor planets that were also observed by the Infrared Astronomical Satellite (IRAS). We have compared the diameters and albedo derived by applying a spherical thermal model to the objects detected by NEOWISE and find that they are generally in good agreement with the IRAS values. We have shown that diameters computed from NEOWISE data are often less systematically biased than those found with IRAS. This demonstrates that the NEOWISE data set can provide accurate physical parameters for the $>157,000$ minor planets that were detected by NEOWISE.
\end{abstract}

Key words: atlases - catalogs - infrared: general - minor planets, asteroids: general

Online-only material: color figures

\section{INTRODUCTION}

Understanding the formation and evolution of the minor planets in our solar system requires good knowledge of the physical parameters that describe each population of asteroids and comets. Models of asteroid migration, for example, are critically dependent on measurements of number counts, sizes, and albedos. Visible light surveys have supplied most of the discoveries of asteroids and comets to date; however, these surveys tend to be biased against low albedo objects and so they are underrepresented in number counts. Furthermore, diameters derived from visible light observations are highly uncertain due to their linear dependence on albedo, which can vary by an order of magnitude for many types of asteroids. Radar observations, stellar occultations, and in situ spacecraft imaging provide powerful means of obtaining precise diameter measurements, but these observations are limited to a small subset of the $\sim 500,000$ minor planets known to exist today. While diameter and albedo can be measured more precisely than with visible light by applying radiometric models to objects for which thermal infrared data have been obtained, the size and albedo distributions obtained in this way will still be biased if the underlying source population was discovered by visible light surveys. In order to mitigate against these biases, it is necessary to undertake a survey that is capable of independent detection and discovery of asteroids at thermal infrared wavelengths. Yet the physical parameters such as size and albedo that are derived from such a new survey must be checked against other wellcharacterized data sets to ensure their reliability.

The Wide-field Infrared Survey Explorer (WISE) is a NASA Medium-class Explorer mission designed to survey the entire sky in four infrared wavelengths: 3.4, 4.6, 12, and $22 \mu \mathrm{m}$ (denoted $W 1, W 2, W 3$, and $W 4$, respectively; Wright et al. 2010; Liu et al. 2008; Mainzer et al. 2005). The final mission data products are a multi-epoch image atlas and source catalogs that will serve as an important legacy for future research. The portion of the pipeline dedicated to finding minor planets (called
NEOWISE) has yielded observations of over 157,000 minor planets, including near-Earth objects, Main Belt Asteroids, comets, Hildas, Trojans, Centaurs, and scattered disk objects (Mainzer et al. 2011a). This represents an improvement of nearly two orders of magnitude more objects observed than WISE's predecessor mission, the Infrared Astronomical Satellite (IRAS; Tedesco et al. 1988; Tedesco 1992; Matson 1986). The WISE survey began on 2010 January 14 and the mission exhausted its primary tank cryogen on 2010 August 5. Exhaustion of the secondary tank and the start of the NEOWISE Post-Cryogenic Mission occurred on 2010 October 1, and the survey ended on 2011 January 31.

In Mainzer et al. (2011b), we demonstrated that thermal models created for minor planets using the WISE/NEOWISE data set produce results that are in good agreement with diameters independently measured by radar, stellar occultations, and in situ spacecraft imaging. By taking the previously measured diameters from methods derived independently of infrared thermal model assumptions, we were able to verify the accuracy of the color corrections given in Wright et al. (2010) for lowtemperature objects such as asteroids. We used the previously measured diameters combined with the Wright et al. (2010) color corrections in conjunction with a faceted spherical thermal model based on the Near-Earth Asteroid Thermal Model (NEATM; Harris 1998) to predict the WISE magnitudes for $\sim 50$ asteroids; these were compared with the observed WISE magnitudes. We found that there were no systematic offsets between predicted and observed magnitudes for these objects, indicating that the color corrections given in Wright et al. (2010) adequately describe the system response. For objects with WISE measurements in two or more bands with good signal to noise (for which the beaming parameter $\eta$ can be fit), we found that diameters can be determined to within $\pm 10 \%$, and visible albedo $p_{V}$ to within $\pm 20 \%$.

In this Letter, we compare diameters and $p_{V}$ obtained with WISE/NEOWISE observations for 1742 asteroids to those determined by IRAS and find that they are generally in good 

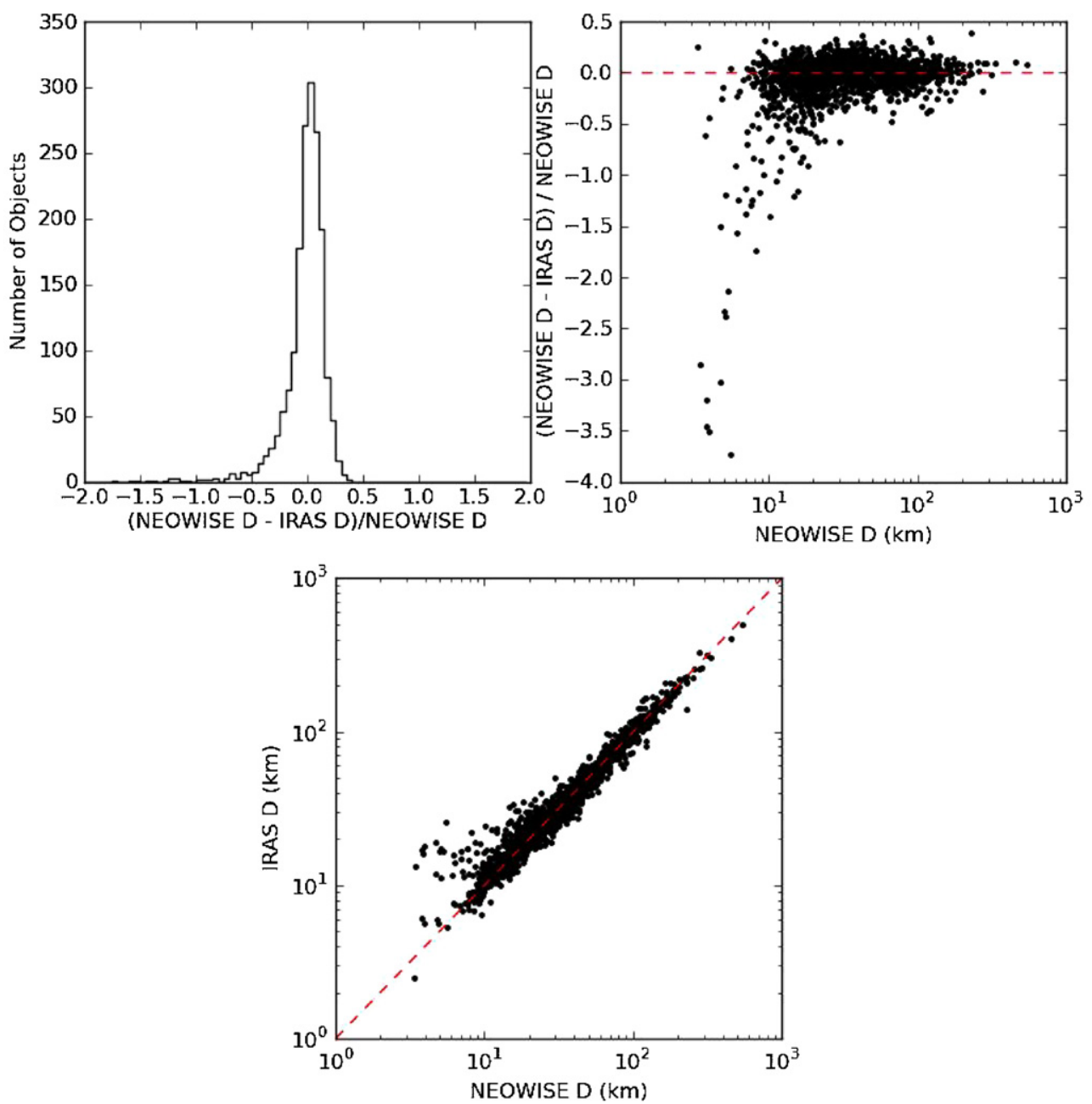

Figure 1. Comparison of the difference in diameters given by Tedesco et al. (2004a) and the diameters found by applying a spherical NEATM model to NEOWISE measurements for 1742 asteroids. It can be seen that the values from Tedesco et al. (2004a) tend to be slightly smaller than NEOWISE-derived diameters. The NEOWISE diameters diverge widely from IRAS for the smallest objects; however, these objects are observed by NEOWISE with high signal-to-noise ratio.

(A color version of this figure is available in the online journal.)

agreement with some slight systematic differences. This work, combined with Mainzer et al. (2011b), demonstrates that the NEOWISE observations of minor planets will produce good physical parameters that will enable a wide range of scientific investigations.

\section{OBSERVATIONS}

We have assembled a list of objects that were observed by IRAS that NEOWISE detected during the fully cryogenic portion of its mission. Of the $\sim 2200$ asteroids observed by IRAS (Tedesco et al. 1988; Tedesco 1992; Matson 1986), we identified NEOWISE detections for 1742 objects. The observations of these objects were retrieved by querying the Minor Planet Center's (MPC) observation files to look for all instances of individual WISE detections of the desired objects that were reported using the WISE Moving Object Processing System (WMOPS; Mainzer et al. 2011a). The resulting set of position/ time pairs were used as the basis of a query of WISE source detections in individual exposures (also known as "Level 1b" images) using the Infrared Science Archive. In order to ensure that only observations of the desired moving object were returned from the query, the search radius was restricted to
0.3 arcsec from the position listed in the MPC observation file. Additionally, since WISE collected a single exposure every $11 \mathrm{~s}$ and observes each part of the sky an average of 10 times, the modified Julian date was required to be within $2 \mathrm{~s}$ of the time specified by the MPC. The following flag values were allowed: cc_flags $=0, \mathrm{P}$, or p and ph_qual $=\mathrm{A}, \mathrm{B}$, or C. Objects brighter than $W 3=4$ and $W 4=0$ magnitudes were assumed to have flux errors equivalent to $0.2 \mathrm{mag}$ due to changes to the shape of the point-spread function as the objects became saturated, and a linear correction was applied to the $W 3$ magnitudes in this brightness regime (the WISE Explanatory Supplement contains a more detailed explanation). As per the Explanatory Supplement (Cutri et al. 2011), objects brighter than $W 3=-2$ and $W 4=-6$ were not used. Each object had to be observed a minimum of three times in at least one WISE band, and it had to be detected at least $40 \%$ of the time when compared to the band with the maximum number of detections (usually, though not always, W3). The WMOPS system is designed to reject inertially fixed objects such as stars and galaxies in bands $W 3$ and $W 4$. Nonetheless, the individual images at all wavelengths were compared with WISE atlas coadd and daily coadd source lists to ensure that inertially fixed sources such as stars and galaxies were not coincident with the moving object detections. 

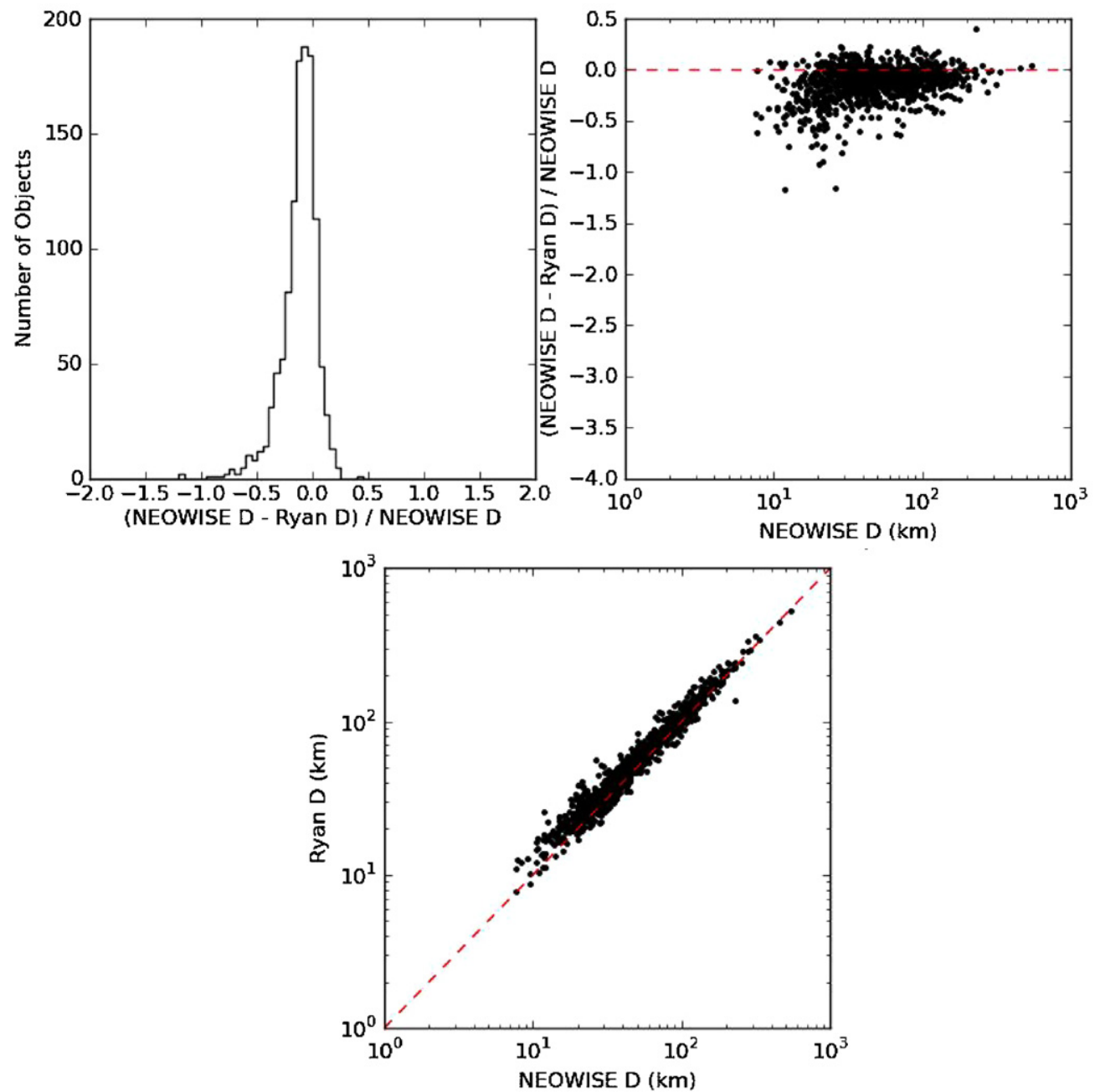

Figure 2. Comparison of the difference in diameters given by Ryan \& Woodward (2010) and the diameters found by applying a spherical NEATM model to NEOWISE measurements for 1155 asteroids. The diameters found by Ryan \& Woodward (2010) tend to be larger than those found by NEOWISE.

(A color version of this figure is available in the online journal.)

This check is particularly important in bands $W 1$ and $W 2$ where the density of background objects (and hence the probability of a blended source) is higher than at longer wavelengths. Any remaining blended sources in bands $W 1$ and $W 2$ were removed. Some objects were observed at multiple epochs, and observations separated by more than three days were modeled separately.

\section{THERMAL MODEL AND REFLECTED SUNLIGHT FITS}

In the Standard Thermal Model (STM) of Lebofsky \& Spencer (1989), the temperature of an asteroid is assumed to be maximum at the subsolar point and zero on the point opposite of this; this is the case of an object with zero thermal inertia. In contrast, in the Fast Rotating Model (FRM; Lebofsky et al. 1978; Veeder et al. 1989; Lebofsky \& Spencer 1989), the asteroid is assumed to be rotating much faster than its cooling time, resulting in a constant surface temperature across all longitudes. The so-called beaming parameter $(\eta)$ was introduced by Lebofsky (1986) in the STM to account for the enhancement of thermal radiation observed at small phase angles. The NEATM of Harris (1998) also uses the beaming parameter $\eta$ to account for cases intermediate between the STM and FRM models. In the STM, $\eta$ is set to 0.756 to match the occultation diameters of (1) Ceres and (2) Pallas, while in the FRM, $\eta$ is equal to $\pi$. With NEATM, $\eta$ is a free parameter that can be fit when two or more infrared bands are available.

We modeled each object as a set of triangular facets covering a spherical surface with diameter equal to the ground-truth measurement (cf. Kaasalainen et al. 2004). The temperature for each facet was computed, and the Wright et al. (2010) color corrections were applied to each facet. The emitted thermal flux for each facet was calculated using NEATM along with the band centers and zero points given in Wright et al. (2010); nightside facets were assumed to contribute no flux. The emissivity, $\epsilon$, was assumed to be 0.9 for all wavelengths (cf. Harris et al. 2009). The objects' absolute magnitudes $(H)$ were taken from Warner et al. (2009) when available; otherwise, the values were taken from the MPC's orbital element files. Unless a direct measurement of $G$ was available from Warner et al. (2009), we assumed a $G$ value of 0.15 . In general, minor planets detected by NEOWISE in bands $W 1$ and $W 2$ contain a mix of reflected sunlight and thermal emission. Thus, it was necessary to incorporate an estimate of reflected sunlight into the thermal model in order to use data from bands $W 1$ and $W 2$. In order to compute the fraction of reflected sunlight in bands $W 1$ and $W 2$, it was also 

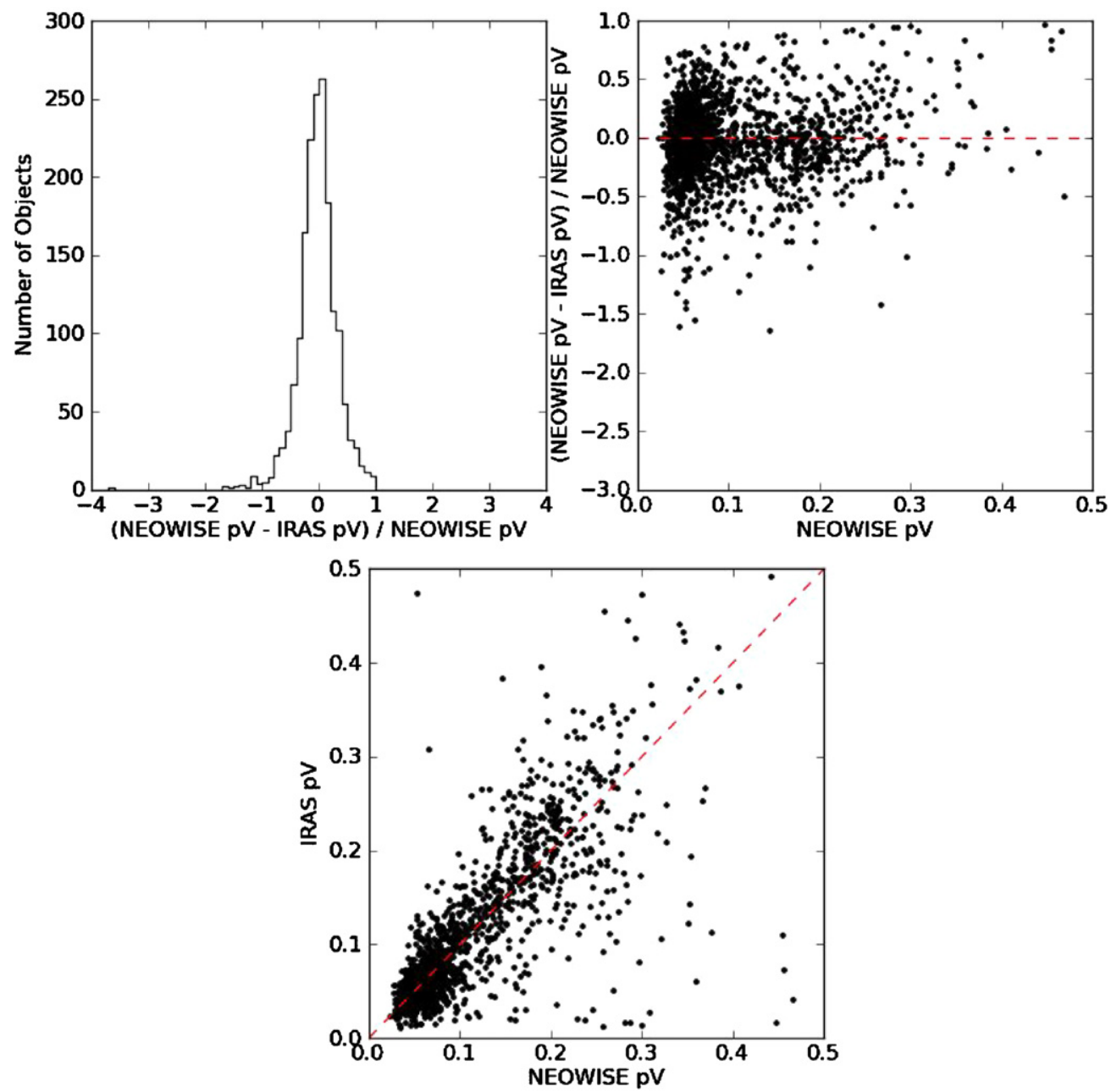

Figure 3. Comparison of the difference in albedos given by Tedesco et al. (2004a) and the diameters found by applying a spherical NEATM model to NEOWISE measurements for 1742 asteroids. It can be seen that the values from Tedesco et al. (2004a) tend to be slightly higher than NEOWISE-derived albedos.

(A color version of this figure is available in the online journal.)

necessary to compute the ratio of the albedo at these wavelengths compared to the visible albedo $\left(p_{\mathrm{IR}} / p_{V}\right)$; the solar spectrum was approximated as a $5778 \mathrm{~K}$ blackbody. As described in Mainzer et al. (2011b), we assumed that $p_{\mathrm{IR}}=p_{3.4 \mu \mathrm{m}}=p_{4.6 \mu \mathrm{m}}$. The flux from reflected sunlight was computed for each WISE band using the IAU phase curve correction of Bowell et al. (1989). Thermal models were computed for each object by grouping together observations having no more than a three-day gap between them.

Error bars on the model magnitudes and subsolar temperatures were determined for each object by running 25 Monte Carlo (MC) trials that varied the objects' $H$ values by 0.3 mag and the WISE magnitudes by their error bars using Gaussian probability distributions. The minimum magnitude error for all WISE measurements fainter than $W 3=4$ and $W 4=3$ magnitudes was $0.03 \mathrm{mag}$, as per the in-band repeatability measured in Wright et al. (2010). For objects brighter than $W 3=4$ and $W 4=3$, the error bars were increased to $0.2 \mathrm{mag}$, as these magnitudes represent the onset of saturation (see the WISE Explanatory Supplement). For those objects for which $\eta$ and $p_{\mathrm{IR}} / p_{V}$ could not be fitted, $\eta$ was set to 1.0 and allowed to vary in the MC trials by 0.25 , and $p_{\mathrm{IR}} / p_{V}$ was set to 1.4 and allowed to vary by 0.5 . These default values were used because they are the mean and standard deviation of $\eta$ and $p_{\mathrm{IR}} / p_{V}$ for the objects for which these parameters could be fit. The error bar for each object's model magnitude was equal to the standard deviation of all the MC trial values.

Although many asteroids are known to be non-spherical, the WISE observations generally consisted of $\sim 10-12$ observations per object uniformly distributed over $\sim 36 \mathrm{hr}$ (Wright et al. 2010; Mainzer et al. 2011a), so on average, a wide range of rotational phases was sampled. Although the variation in effective spherical diameter resulting from rotational effects tends to be averaged out, caution must be exercised when interpreting effective diameter results using spherical models for objects that are known to have large-amplitude light curve variations. In our sample, 179 objects have NEOWISE observations at multiple epochs (meaning that the groups of observations were separated by more than 10 days). Of these, all but 24 had diameters that agreed to within $10 \%$, and most of the remaining objects had $W 3$ peak-to-peak amplitudes $>0.3 \mathrm{mag}$, indicating that they are likely to be non-spherical. Non-spherical objects observed at different viewing geometries can lead to different diameters when using a spherical model. However, since $87 \%$ of the objects with multi-epoch observations have diameters that agree to within $10 \%$, we conclude that multiple observational epochs do not contribute significantly to the differences observed between IRAS and NEOWISE diameters. 

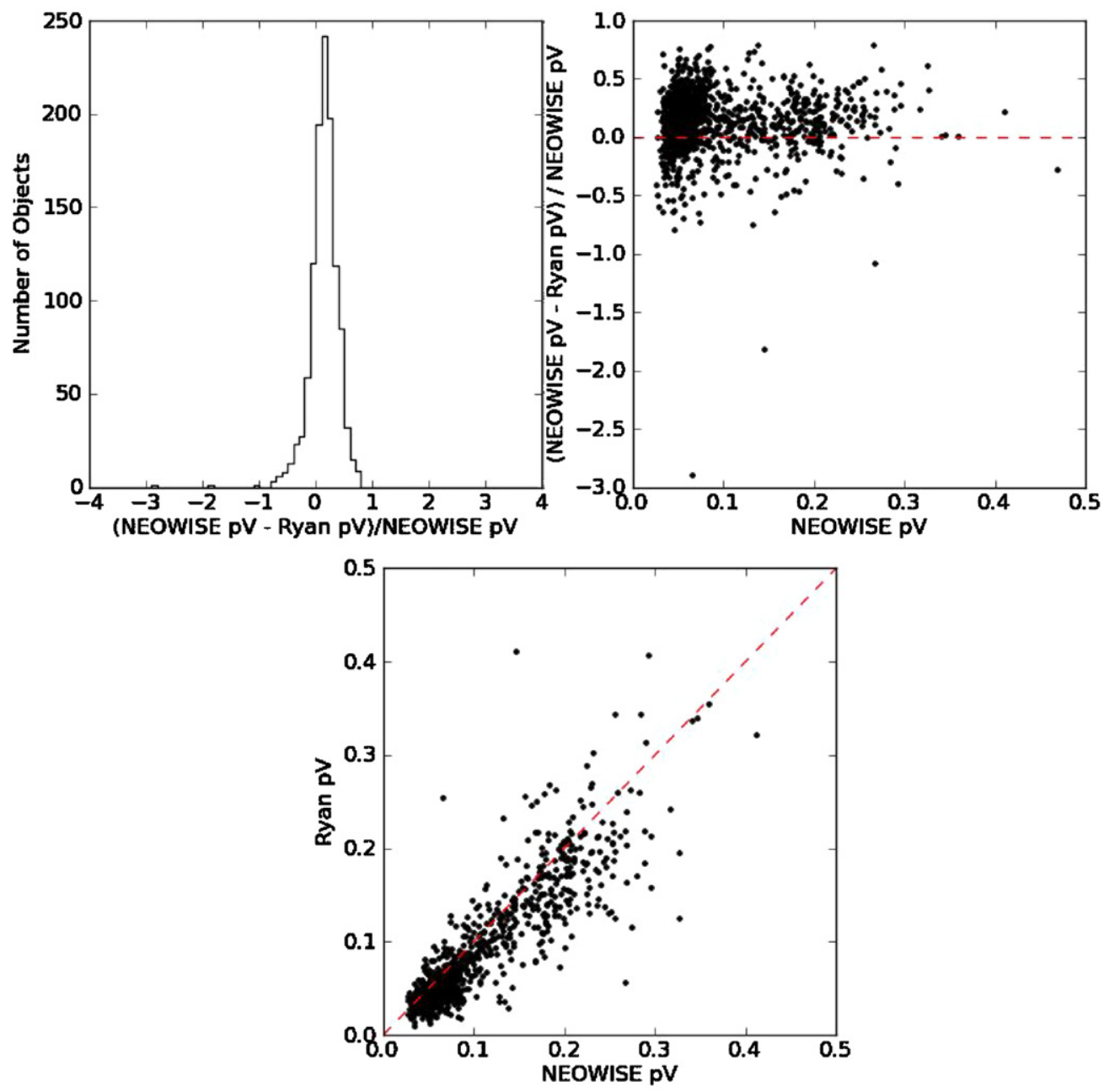

Figure 4. Comparison of the difference in albedos given by Ryan \& Woodward (2010) and the albedos found by applying a spherical NEATM model to NEOWISE measurements for 1155 asteroids. The albedos found by Ryan \& Woodward (2010) tend to be lower than those found by NEOWISE.

(A color version of this figure is available in the online journal.)

\section{RESULTS}

Tedesco et al. (2002) reported observations of $\sim 2200$ asteroids within the Supplemental IRAS Minor Planet Survey (SIMPS). The diameters and albedos in that work were computed using the STM. Similar to the work of Walker (2003), who computed NEATM diameters and albedos for 654 asteroids using IRAS fluxes, Ryan \& Woodward (2010) applied a spherical NEATM model to $\sim 1500$ asteroids found in the SIMPS and Mid-Course Space Experiment (Tedesco et al. 2004b) data. Figures 1 and 2 show the comparison between diameters found using NEOWISE observations and those given by Tedesco et al. (2004a) and Ryan \& Woodward (2010), respectively. Figures 3 and 4 show the comparison between NEOWISE and Tedesco/ Ryan albedos. The diameters given by Tedesco et al. (2004a) are systematically lower than the NEOWISE diameters, while the diameters computed by Ryan \& Woodward (2010) are systematically higher. Also, $p_{V}$ given by Tedesco et al. (2004a) is systematically higher than the NEOWISE $p_{V}$ values, but the Ryan \& Woodward (2010) values tend to be slightly lower. In Figure 5, we show the diameters of Tedesco et al. (2004a) and Ryan \& Woodward (2010) compared to the diameters of 78 objects, with diameters measured by radar, occultation, or spacecraft imaging (see Mainzer et al. 2011b for a complete list of references).
These objects have been selected to have $W 3$ peak-to-peak amplitudes $<0.5$ mag to avoid the worst complications caused by applying spherical models to highly elongated objects. As can be seen in the figure, the Tedesco et al. (2004a) diameters are skewed toward slightly smaller sizes than the radar, etc., measurements, whereas the Ryan \& Woodward (2010) diameters are biased toward larger sizes. We propose that these biases are caused by the band-to-band color corrections derived for IRAS. As described in Tedesco et al. (2002), band-to-band corrections were derived by requiring that the 10 and $20 \mu \mathrm{m} I R A S$ observations simultaneously matched a diameter derived from a stellar occultation of (1) Ceres. Because the STM parameters were set to match Ceres, Tedesco et al. (2002) found a 7\% difference between diameters derived for 13 objects, with diameters measured from stellar occultations. The NEATM model applied by Ryan \& Woodward (2010) does not appear to address this issue. Furthermore, Ryan \& Woodward (2010) applied cutoffs to $\eta$ of 0.75 and 2.75. As described above, in our NEATM models applied to the NEOWISE data, we cut off $\eta$ at the limit predicted by the FRM, $\pi$. In the STM, $\eta$ was set to 0.756 by matching the diameters of Ceres and Pallas; however, there is no reason in principle why it cannot range to lower values. In our application of NEATM, we allow $\eta$ to range arbitrarily low and find no fitted $\eta$ values less than $\sim 0.53$. 

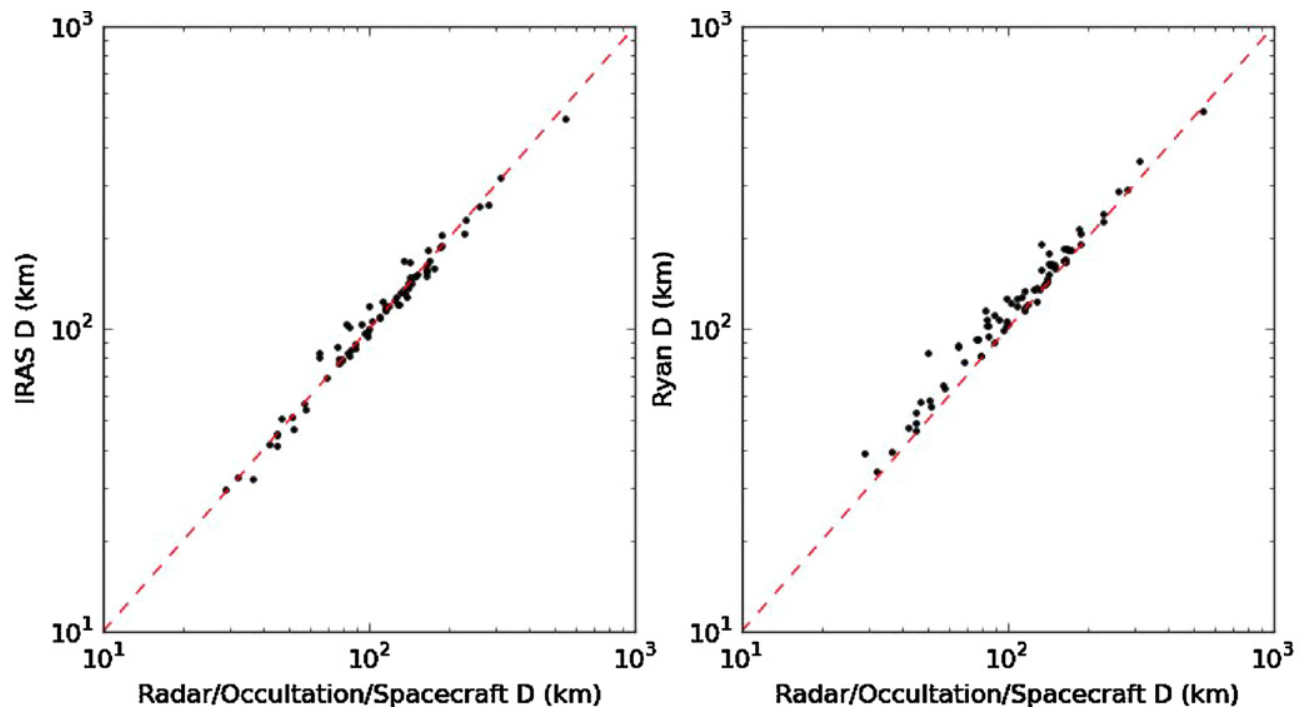

Figure 5. Comparison of diameters found by radar, stellar occultation, or in situ spacecraft imaging for 78 asteroids and the diameters found by Tedesco et al. (2004a) and Ryan \& Woodward (2010). The objects were selected to have $W 3$ peak-to-peak amplitudes $<0.5$. It can be seen that both methods of computing IRAS diameters are somewhat biased when compared to the radar diameters. In Mainzer et al. (2011b), we show that there is no systematic bias found in model and observed WISE magnitudes for a similar set of objects with independently measured diameters.

(A color version of this figure is available in the online journal.)

As described above, in Mainzer et al. (2011b), we used asteroids with previously known diameters and $H$ magnitudes to compare our model and observed WISE magnitudes, and we found no systematic offsets. We conclude that the diameters for minor planets derived from NEOWISE are generally in good agreement with those found by IRAS and are likely more free of systematic biases than the diameters provided in either Tedesco et al. (2002) or Ryan \& Woodward (2010). Together with Mainzer et al. (2011b), this demonstrates that the NEOWISE data set will produce good quality physical parameters for the $>157,000$ minor planets it contains.

This publication makes use of data products from the Widefield Infrared Survey Explorer, which is a joint project of the University of California, Los Angeles, and the Jet Propulsion Laboratory/California Institute of Technology, funded by the National Aeronautics and Space Administration. This publication also makes use of data products from NEOWISE, which is a project of the Jet Propulsion Laboratory/California Institute of Technology, funded by the Planetary Science Division of the National Aeronautics and Space Administration. We gratefully acknowledge the extraordinary services specific to NEOWISE contributed by the International Astronomical Union's Minor Planet Center, operated by the Harvard-Smithsonian Center for Astrophysics, and the Central Bureau for Astronomical Telegrams, operated by Harvard University. We acknowledge use of NASA's Planetary Data System. This research has made use of the NASA/IPAC Infrared Science Archive, which is operated by the Jet Propulsion Laboratory, California Institute of Technology, under contract with the National Aeronautics and Space Administration. This research has made use of NASA's Astrophysics Data System.

\section{REFERENCES}

Bowell, E., Hapke, B., Domingue, D., et al. 1989, in Asteroids II, ed. R. P. Binzel, T. Gehrels, \& M. S. Matthews (A90-27001 10-91; Tucson, AZ: Univ. Arizona Press), 524

Cutri, R. M., Wright, E. W., Conrow, T., et al. 2011, Explanatory Supplement to the WISE Preliminary Data Release Products, http://wise2.ipac. caltech.edu/docs/release/prelim/expsup/wise_prelrel_toc.html

Harris, A. 1998, Icarus, 131, 291

Harris, A., Mueller, M., Lisse, C., \& Cheng, A. 2009, Icarus, 199, 86

Kaasalainen, M., Pravec, P., Krugly, Y., et al. 2004, Icarus, 167, 178

Lebofsky, L., \& Spencer, J. 1989, in Asteroids II, ed. R. P. Binzel, T. Gehrels, \& M. S. Matthews (Tucson, AZ: Univ. Arizona Press), 128

Lebofsky, L. A., Sykes, M. V., Tedesco, E. F., et al. 1986, Icarus, 68, 239

Lebofsky, L., Veeder, G., Lebofsky, M., \& Matson, D. 1978, Icarus, 35, 336

Liu, F., Cutri, R., Greanias, G., et al. 2008, Proc. SPIE, 7017, 16

Mainzer, A., Bauer, J., Grav, T., et al. 2011a, ApJ, 731, 53

Mainzer, A., Eisenhardt, P., Wright, E. L., et al. 2005, Proc. SPIE, 5899, 262

Mainzer, A., Grav, T., Masiero, J., et al. 2011b, ApJ, in press

Matson, D. (ed.) 1986, The IRAS Asteroid and Comet Survey, JPL D-3698 (Pasadena, CA: JPL)

Ryan, E., \& Woodward, C. 2010, AJ, 140, 933

Tedesco, E. F. (ed.) 1992, in IRAS Minor Planet Survey (Phillips Laboratory Technical Report No. PL-TR-92-2049; MA: Hanscom Air Force Base), http://irsa.ipac.caltech.edu/IRASdocs/surveys/minor.html

Tedesco, E., Matson, D., Veeder, G., \& Lebofsky, L. 1988, in Proc. Third IRAS Conf. on Comets to Cosmology, Vol. 297 (Berlin: Springer), 19

Tedesco, E., Noah, P., Noah, M., \& Price, S. 2002, AJ, 123, 1056

Tedesco, E., Noah, P. V., Noah, M., \& Price, S. D. 2004a, IRAS Minor Planet Survey, IRAS-A-FPA-3-RDR-IMPS-V6.0, NASA Planetary Data System

Tedesco, E. T., Egan, M. P., \& Price, S. D. 2004b, MSX Infrared Minor Planet Survey, MSX-A-SPIRIT3-5-SBN0003-MIMPS-V1.0, NASA Planetary Data System

Veeder, G., Hanner, M. S., Matson, D. L., et al. 1989, AJ, 97, 1211

Walker, R. 2003, American Astronomical Society, DPS Meeting 35, 980

Warner, B., Harris, A., \& Pravec, P. 2009, Icarus, 202, 134

Wright, E. L., Eisenhardt, P. R. M., Mainzer, A. K., et al. 2010, AJ, 140, 1868 